\title{
Performing current versus voltage measurements of single-walled carbon nanotubes using scanning force microscopy
}

Cite as: Appl. Phys. Lett. 80, 1462 (2002); https://doi.org/10.1063/1.1453475

Submitted: 23 July 2001 - Accepted: 17 December 2001 • Published Online: 22 February 2002

P. J. de Pablo, C. Gómez-Navarro, M. T. Martínez, et al.

\section{ARTICLES YOU MAY BE INTERESTED IN}

Electrical resistivity of polypyrrole nanotube measured by conductive scanning probe microscope: The role of contact force

Applied Physics Letters 81, 4625 (2002); https://doi.org/10.1063/1.1528281

Nanoscale charge transport in an electroluminescent polymer investigated by conducting atomic force microscopy

Applied Physics Letters 81, 2572 (2002); https://doi.org/10.1063/1.1509464

Conducting probe atomic force microscopy applied to organic conducting blends

Applied Physics Letters 79, 2993 (2001); https://doi.org/10.1063/1.1413717

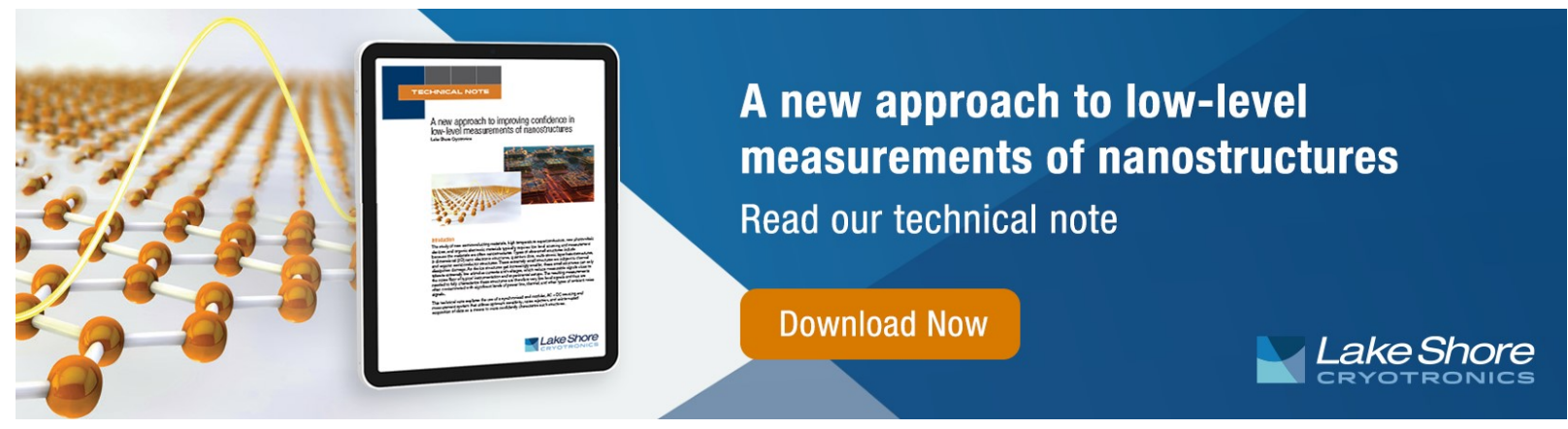




\title{
Performing current versus voltage measurements of single-walled carbon nanotubes using scanning force microscopy
}

\author{
P. J. de Pablo and C. Gómez-Navarro \\ Au-Queried Laboratorio de Nuevas Microscopías, Departamento de Física de la Materia Condensada, \\ Universidad Autónoma de Madrid, E-28049 Madrid, Spain
}

M. T. Martínez, A. M. Benito, and W. K. Maser

Instituto de Carboquímica, CSIC, Miguel Luesma Castán 4, E-50015 Zaragoza, Spain

J. Colchero, J. Gómez-Herrero, ${ }^{\text {a) }}$ and A. M. Baró

Laboratorio de Nuevas Microscopías, Departamento de Física de la Materia Condensada, Universidad Autónoma de Madrid, E-28049 Madrid, Spain

(Received 23 July 2001; accepted for publication 17 December 2001)

\begin{abstract}
In this letter, a method is presented to perform current versus voltage measurements on carbon nanotubes using the tip of a scanning force microscope as an electrode that can be positioned along the molecule. This method allows current versus voltage measurements to be carried out at any spot along a nanotube. By using this method, we present indications of ballistic transport in carbon nanotubes, as well as effects in the electrical properties due to the mechanical deformation. This is a general technique that can be applied to any conducting nanowire. (C) 2002 American Institute of Physics. [DOI: 10.1063/1.1453475]
\end{abstract}

Over the past few years there has been increasing interest for molecular wires, ${ }^{1}$ and in particular for carbon nanotubes. ${ }^{2}$ A carbon nanotube is a graphene layer rolled up forming a tube of nanometer size. They were found to be good electrical conductors with astonishing properties, showing ballistic conductance, ${ }^{3}$ superconducting behavior, ${ }^{4}$ and Aharonov-Bohm effect. ${ }^{5}$ Carbon nanotubes have also been used as field-emission emitters, ${ }^{6}$ chemical detectors, ${ }^{7}$ nanotweezers, ${ }^{8}$ etc. In these experiments ${ }^{9,10}$ a single walled carbon nanotube (SWNT) is placed between two fixed electrodes and the electrical current flowing through it was measured. In this way, current versus voltage $(I-V)$ curves cannot be carried out along the length of a carbon nanotube. Measuring $I-V$ curves on several spots along the SWNT would make it possible to check fundamental electrical properties of these nanowires, such as ballistic conduction, the influence of mechanical defects on the transport properties or the quality of the metallic electrode-nanotube contact. ${ }^{11}$

In our setup we extend previous experiments, where the conductivity of multiwalled carbon nanotubes is checked at a fixed voltage by using a conductive scanning force microscope (SFM) tip without carrying out $I-V$ measurements. ${ }^{12}$ In another work, a scanning tunneling microscopy tip slides along a SWNT, while measuring $I-V$ curves, ${ }^{13}$ but without imaging the nanotube used in the experiment. With the method presented in this letter, it is possible to acquire $I-V$ curves along the nanotube and to image the corresponding molecule.

Samples are prepared by adsorbing SWNT on $\mathrm{SiO}_{2}{ }^{14}$ substrates. After the molecules are deposited, gold is thermally evaporated on the sample using a masking technique. The final result is a sample with two gold electrodes separated by a gap $(\sim 5 \mu \mathrm{m})$ free of gold with a serendipitous

${ }^{a)}$ Electronic mail: julio.gomez@uam.es distribution of molecules. Some of them are partially covered with gold. A SFM is used in noncontact dynamic mode ${ }^{15}$ using a Nanotec Electrónica system. ${ }^{16}$ This mode is not required to image multiwalled carbon nanotubes, ${ }^{12}$ since these are more strongly anchored to the surface than SWNT. ${ }^{17}$ However, we have observed that SWNT can be displaced when using a contact scanning mode. To perform electrical measurements we use Olympus cantilevers with $80 \mathrm{kHz}$ resonance frequency consecutively covered with titanium and gold. By inspection, a SWNT is selected to perform the experiments $^{18}$ (Fig. 1). The tip is then approached to a previously chosen spot on the selected SWNT, making simultaneous electrical and mechanical contact. ${ }^{19}$ At the maximum tip indentation a $I-V$ curve is acquired. ${ }^{20}$ In this way, the loading force applied to the contact is selected prior to the acquisition of the $I-V$ data. Moreover, the details of $I-V$ acquisition can also be conveniently selected.

In order to assess whether the resistance and shape of the

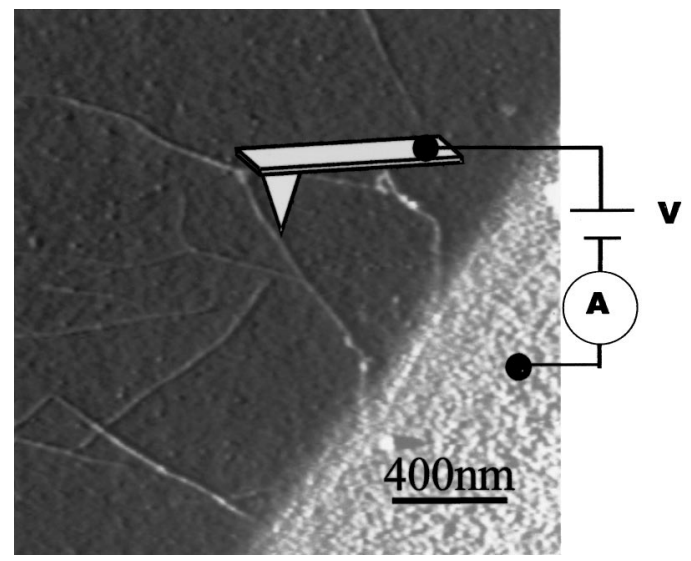

FIG. 1. Experimental setup. Schematic representation of the circuit used in the experiments. The $I-V$ curves are taken by contacting a metal-covered tip and a SWNT partially covered with gold. 


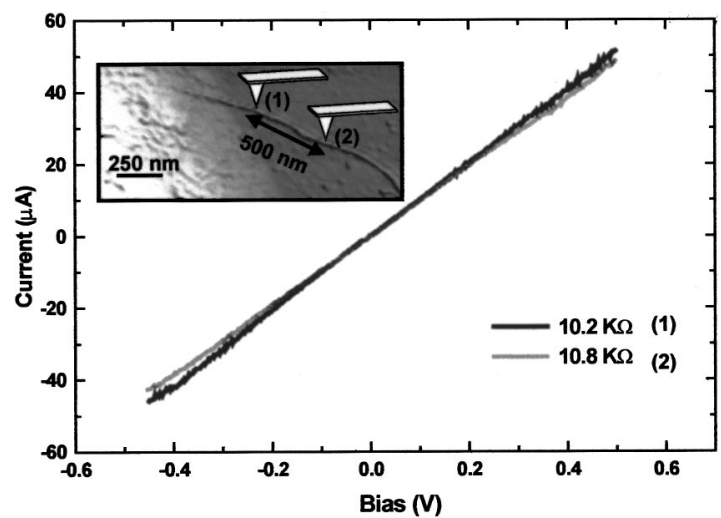

FIG. 2. Linear $I-V$ curves characteristics with $\sim 10 \mathrm{k} \Omega$ resistance on a 2.3 $\mathrm{nm}$ diameter tube depicted in the inset. The distance between $I-V$ curves is about $500 \mathrm{~nm}$.

curve is affected by the tip-nanotube contact, one nanotube is used as reference sample to verify that the tip is not modified during the experiments. After every experiment we return to the reference nanotube to control that the same $I-V$ curve is obtained.

With this method the total resistance of a tube can be separated in two contributions: the contact resistance (gold electrode + SFM tip) and the intrinsic resistance from the SWNT itself. In this way information regarding the transport mechanism of the tube (ballistic, diffusive, etc.) can be obtained. While a large number of tubes present a total resistance greater than $\sim 100 \mathrm{k} \Omega$, we found some nanotubes with lower resistance where ballistic transport is clearly present. For instance, we have measured $I-V$ curves at two different spots on the $2.3 \mathrm{~nm}$ diameter SWNT of the inset in Fig. 2. This figure also shows the corresponding $I-V$ curve from which a resistance of $\sim 10 \mathrm{k} \Omega$ is obtained. Moreover, we can relate the measured resistance to a transmission probability using the Landauer formula $R_{j}=\left(h / 4 e^{2}\right)\left(1-T_{j}\right) / T_{j}$, which expresses the resistance per nanotube, ${ }^{1,21} T_{j}$ being the transmission coefficient for electrons along the length of the nanotube. Using the data in Fig. 2 we find that $T_{j}=0.91$ for one single nanotube and $T_{j}=0.78$ assuming three conducting SWNTs forming a rope. ${ }^{22}$ Taking the distance between both spots into account, the coherence length is greater than $5 \mu \mathrm{m}$ in the first case and $1.5 \mu \mathrm{m}$ in the second case. Considering only one nanotube, the coherence length is three times greater than the one reported by Bachtold et al. ${ }^{21}$

As an additional example of the method described here we have measured the conductance of a SWNT that had been mechanically deformed in order to investigate the influence of nanotube deformation on its electrical properties. As discussed above, contact experiments with the SFM tip may produce modifications of the SWNT shape. Figure 3(a) is a topographic image of a SWNT showing one of these modifications at the point marked D. While the differential resistance before point D saturates at large bias voltages, after point D it decreases with bias [see Figs. 3(a) and 3(b)]. Therefore, we conclude that by modifying the SWNT geometry, changes in the electrical transport properties of the nanotubes can be induced. Again we extend a previous result where changes in resistance are induced by local deformations using a SFM tip. ${ }^{23}$ Here we do not only carry out resistance measurements, but complete $I-V$ curves. Thus, we
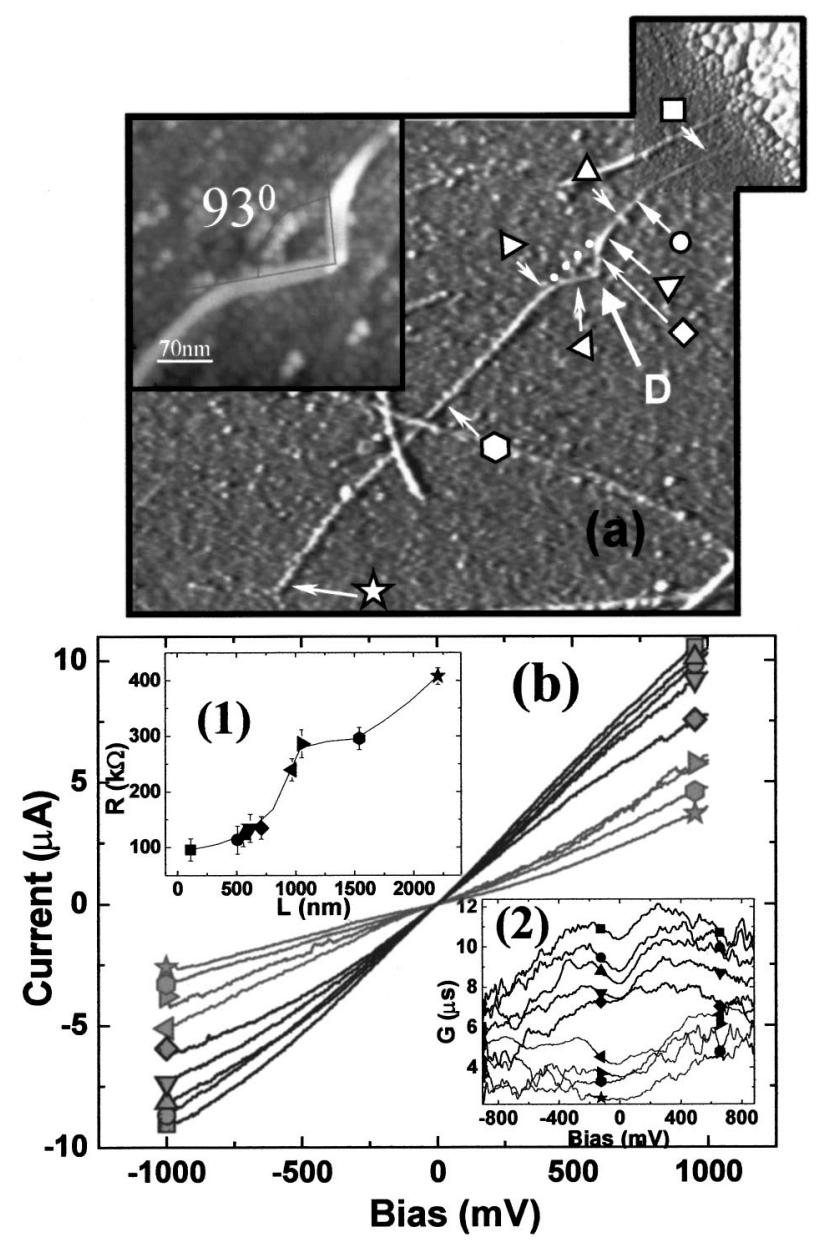

FIG. 3. SWNT deformation performed during a contact experiment with the SFM tip. In (a) the SWNT image with the deformation "D" is shown. The white dots show the previous SWNT shape. Note the gold electrode in the upper right corner. The spots where $I-V$ curves have been acquired are marked conveniently. In (b) the $I-V$. curves performed before deformation $\mathrm{D}$ are plotted in black and the $I-V$ performed in places of the nanotube after $\mathrm{D}$ are plotted in gray color. The symbols localize the $I-V$ curves in the places marked in (a). In the inset (1) the resistance near $0 \mathrm{~V}$ along the nanotube is shown, with the corresponding symbols. Finally, in the inset (2), the differential conductance is depicted with the same prior color and symbol codes.

can determine how the electrical characteristic of the nanotube varies due to the deformation.

In our experiment, the $I-V$ curves change from linear around zero bias [dark lines in Fig. 3(b)] before the deformation D to nonlinear [gray lines in Fig. 3(b)] after the deformation D. In the inset (2) of Fig. 3(b) the differential conductance of all the $I-V$ curves of Fig. 3(b) taken after modification $\mathrm{D}$ decreases at low voltages. The essential characteristic of the $I-V$ curves vary from saturated before deformation to nonsaturated after it. This suggests that the saturation of $I-V$ is not related to the contact resistance, ${ }^{24}$ but to the intrinsic structure of the nanotube. In addition, in inset (1) of Fig. 3(b) the behavior of the resistance around zero bias is presented, showing a sudden increase of the resistance precisely at the deformation point $\mathrm{D}$.

Changes of the transport properties of the SWNT have been predicted in terms of changes in the carbon-carbon bonds. ${ }^{25}$ To produce these changes, mechanical stress has to be applied within the atomic structure of the SWNT. The critical curvature $K_{c}$ at which a SWNT buckles is given by 
$(0.155 \mathrm{~nm}) d^{-2},{ }^{26}$ being $d$ the diameter of the tube. In our case, with $2 \mathrm{~nm}$ diameter, we obtain $K_{c} \geqslant 0.05 \mathrm{~nm}^{-1}$. Figure 3(a) shows an enlarged view of a modification induced by the tip. From this image, a minimum curvature of $K \geqslant 0.06 \mathrm{~nm}^{-1}$ is obtained. Since $K>K_{c}$ the tube is buckled at $\mathrm{D}$, indicating that a large mechanical stress has been stored at this point, locally buckling the nanotube.

It has been shown that with a stress $\sigma \approx 100 \mathrm{GPa}$ dislocations in the carbon-carbon bonds are induced, ${ }^{27}$ locally changing the pattern of the bonds from hexagons to pentagons. This plastic deformation varies the electronic properties. It has been theoretically predicted that this kind of defect can open a gap in nanotubes with indexes $(n, m+3 i) .{ }^{28}$ For example, in the metallic nanotube $(9,0)$ by inducing one of these local modifications a gap of $0.5 \mathrm{eV}$ is opened. Using the measured curvature radius at $D(r \approx 15 \mathrm{~nm})$ a stress $\sigma$ $\approx 80 \mathrm{GPa}$ is found, ${ }^{29}$ very close to the predicted critical $\operatorname{stress}^{27}(\sigma \approx 100 \mathrm{GPa})$. In addition, other mechanisms have been proposed to account for electronic transport changes due to elastic deformations. ${ }^{30}$ This mechanism would involve mixing of $\sigma$ and $\pi$ orbitals.

In accordance with these theoretical predictions we interpret our results as the observation of the transition from a metallic to a semiconducting state due to the creation of a local dislocation in the tube. We note that these defects do not always produce a change in the shape of the $I-V$ curves measured on the SWNT. In general, these $I-V$ curves remain essentially unmodified and only the electronic properties of the tube change locally at the deformation point. This is consistent with theoretical calculations, which indicate that mechanical distortions of tubes do not significantly affect the conductance, unless the strain is great enough to produce local plastic deformations of the carbon bonds of the nanotube.

In summary, we have presented a method to perform $I-V$ curves along a single SWNT with a SFM setup. This method has been applied to check the ballistic transport of SWNT, finding transmission coefficients of about 0.9. The effect of applying stresses to a SWNT has been also investigated. We find that the electrical properties of such SWNT can be dramatically affected. In particular, the shape of the $I-V$ curves may vary from linear to semiconductor, before and after the place of the deformation point, respectively.

The authors acknowledge fruitful discussions with P. A. Serena and J. J. Sáenz and to the Comunidad de Madrid for financial support through Project No. 07N/0024/1998 and to Ministerio de Educación y Ciencia through Project No. PB95-0169.
${ }^{1}$ S. Datta, Electron Transport in Mesoscopic Systems (Cambridge University Press, Cambridge, UK, 1995).

${ }^{2}$ S. Iijima, Nature (London) 354, 56 (1991).

${ }^{3}$ S. Frank, P. Poncharal, Z. L. Wang, and W. A. de Heer, Science 280, 1744 (1998).

${ }^{4}$ A. Y. Kasumov, M. Kociak, S. Guéron, B. Reulet, V. T. Volkov, D. V. Klinov, and H. Bouchiat, Science 284, 1508 (1999).

${ }^{5}$ A. Bachtold, C. Strunk, J-P. Salvetat, J.-M. Bonard, L. Forró, T. Nussbaumer, and C. Schönenberger, Nature (London) 397, 673 (1999).

${ }^{6}$ D. Lovall, M. Buss, E. Graugnard, R. P. Andres, and R. Reifenberger, Phys. Rev. B 61, 5683 (2000).

${ }^{7}$ S. S. Wong, E. Joselevisch, A. T. Woolley, C. L. Cheung, and C. M. Lieber, Nature (London) 394, 52 (1998).

${ }^{8}$ P. Kim and C. M. Lieber, Science 286, 2148 (1999).

${ }^{9}$ E. W. Wong, P. E. Sheehan, and C. M. Lieber, Science 393, 49 (1997).

${ }^{10}$ S. J. Tans, A. R. M. Verschueren, and C. Dekker, Nature (London) 393, 49 (1998).

${ }^{11}$ M. P. Anantram, Appl. Phys. Lett. 78, 2055 (2001).

${ }^{12}$ H. Dai, E. W. Wong, and Ch. M. Lieber, Science 272, 523 (1996).

${ }^{13}$ P. G. Collins, A. Zettl, H. Bando, A. Thess, and R. E. Smalley, Science 278, 100 (1997).

${ }^{14}$ M. Burghard, G. Duesberg, G. Philipp, J. Muster, and S. Roth, Adv. Mater. 10, 584 (1998).

${ }^{15}$ P. J. de Pablo, J. Colchero, M. Luna, J. Gómez-Herrero, and A. M. Baró, Phys. Rev. B 61, 14179 (2000).

${ }^{16}$ Nanotec Electrónica, C/ Padilla 1, Madrid, Spain. www.nanotec.es

${ }^{17}$ T. Hertel, R. E. Walkup, and P. Avouris, Phys. Rev. B 58, 13870 (1998).

${ }^{18}$ We should notice that with a SFM it is impossible to determine whether the sample selected contains one or several SWNTs. See, for example, J. Muster, G. S. Duesberg, S. Roth, and M. Burghard, Appl. Phys. A: Mater. Sci. Process. 69, 261 (1999); J. S. Villarrubia, J. Res. Natl. Inst. Stand. Technol. 102, 425 (1997).

${ }^{19}$ P. J. de Pablo, M. T. Martínez, J. Colchero, J. Gómez-Herrero, W. K. Maser, A. M. Benito, E. Muñoz, and A. M. Baró, Adv. Mater. 12, 573 (2000).

${ }^{20} \mathrm{An}$ amplifier SR750 to register the current flowing through the nanotube is used.

${ }^{21}$ A. Bachtold, M. S. Fuhrer, S. Plyasunov, M. Forero, E. H. Anderson, A. Zettl, and P. L. McEuen, Phys. Rev. Lett. 84, 6082 (2000).

${ }^{22}$ Taking into account $\mathrm{n}$ nanotubes in the rope, the transmission coefficient $T$ yields $T(n)=\left[n\left(\Delta R / R_{0}\right)+1\right]^{-1}$, where $\Delta R$ is the increment of resistance between two points in the nanotube, and $R_{0}=h / 4 e^{2} \approx 6.5 \mathrm{k} \Omega$

${ }^{23}$ T. Tombler, C. Zhou, L. Alexeyev, J. Konk, H. Dai, L. Liu, C. S. Jayanthi, M. Tang, and S. Y. Wu, Nature (London) 405, 769 (2000); D. Bozovic, M. Bockrath, J. H. Hafner, C. M. Lieber, H. Park, and M. Tinkham, Appl. Phys. Lett. 78, 3693 (2001).

${ }^{24}$ Z. Yao, C. L. Kane, and C. Dekker, Phys. Rev. Lett. 84, 2941 (2000).

${ }^{25}$ L. Chico, V. H. Crespi, L. X. Benedict, S. G. Louie, and M. L. Cohen, Phys. Rev. Lett. 76, 971 (1996).

${ }^{26}$ B. I. Yakobson, C. J. Brabec, and J. Bernhol, Phys. Rev. Lett. 76, 2511 (1996).

${ }^{27}$ P. Zhang, P. E. Lammert, and V. H. Crespi, Phys. Rev. Lett. 81, 5346 (1998).

${ }^{28}$ V. H. Crespi, M. L. Cohen, and A. Rubio, Phys. Rev. Lett. 79, 2093 (1997).

${ }^{29}$ For a cylinder of radius $R$, the stress $\sigma$ originated by a local buckling of radius $r$ is given by $\sigma=(r / R) \times E$. $E$ is the Young modulus of the carbon nanotube, which is taken as $1.2 \mathrm{TPa}$ (Ref. 23).

${ }^{30}$ A. Rochefort, P. Avouris, F. Lesage, and D. R. Salahub, Phys. Rev. B 60, 13824 (1999); P. Avouris, T. Hertel, R. Martel, T. Schmidt, H. R. Shea, and R. E. Walkup, Appl. Surf. Sci. 141, 201 (1999). 